INVESTIGATIVE UROLOGY

\title{
Protective effects of cranberries on infection-induced oxidative renal damage in a rabbit model of vesico-ureteric reflux
}

Han CH, Kim SH, Kang SH, Shin OR, Lee HK, Kim HJ, Cho YH

Department of Urology, College of Medicine, the Catholic University of Korea, Seoul, Korea

BJU Int. 2007; 100: 1172-5

Objective: To evaluate the protective effects of cranberry fruit, which have known antioxidant effects, on infection-induced oxidative renal damage in a rabbit model of vesico-ureteric reflux (VUR).

Materials and Methods: In all, 36 New Zealand male rabbits were divided into five groups, with a sham operation in four rabbits serving as the control (group 1). To create unilateral VUR the roof of the left intravesical ureter was incised, and VUR confirmed 2 weeks after surgery. In all, 32 rabbits with VUR were divided into four groups; 2, VUR alone (with sterile urine); 3, a group infected with Escherichia coli; 4, with intravesical E. coli instillation but fed cranberries; and 5, intravesical E. coli instillation plus an intraperitoneal injection with melatonin group. At 3 weeks after surgery the rabbits were killed, the kidneys obtained and examined histopathologically to evaluate inflammation, fibrosis and tubular changes. Oxidative renal damage was evaluated by measuring malondialdehyde in the renal tissue.

Results: Grossly, the refluxing kidney was larger than the contralateral normal kidney, and the refluxing ureter was dilated and tortuous. Microscopy of tissues from the kidneys in group 3 showed apparent periglomerular mononuclear cell infiltration, tubular dilatation and atrophy, and interstitial fibrosis. The kidneys from groups 2,4 and 5 showed mild mononuclear cell infiltration with no interstitial fibrosis. The level of malondialdehyde in the 
kidneys of group 3 was significantly higher than that in group 2, 4 and $5(\mathrm{P}<0.05)$; the level in groups 4 and 5 did not differ significantly from that in group 2.

Conclusions: This study shows that cranberries have an anti-inflammatory effect through their antioxidant function and might prevent infection-induced oxidative renal damage. Thus, clinically cranberries might be used as a beneficial adjuvant treatment to prevent damage due to pyelonephritis in children with VUR.

\section{Editorial Comment}

The cranberry, a fruit with antioxidant properties, has been used for preventing urinary tract infections. Cranberry juice is also known to have activity against oxygen free radicals, which are produced during infection and are important for promoting renal damage. The authors evaluated the protective effects of cranberries on infection-induced oxidative renal damage in rabbits with experimental vesico-ureteral reflux.

It was demonstrated that melatonin and cranberry powder decreased inflammation and the accumulation of malondialdehyde in the kidney, which suggests that cranberry compounds act as an antioxidant as well as an anti-adherent in preventing infection-induced renal damage.

Dr. Francisco J.B. Sampaio

Full-Professor and Chair, Urogenital Research Unit

State University of Rio de Janeiro

Rio de Janeiro, RJ, Brazil

E-mail: sampaio@urogenitalresearch.org

\section{Proportional analysis of pig kidney arterial segments: differences from the human kidney}

Pereira-Sampaio M, Favorito LA, Henry R, Sampaio FJ

Department of Morphology, Fluminense Federal University, Niteroi, Brazil

J Endourol. 2007; 21: 784-8

Purpose: To present a systematic study and a proportional analysis of the arterial segments of the pig kidney. Materials and Methods: Sixty-one three-dimensional endocasts of the arterial segments of pig kidneys were studied. Each segment was injected with a resin of a different color. Cavalieri's principle was used to calculate the volume of each renal segment, and these results were compared with the results from the point-counting planimetry method used on photographs of pig-kidney surfaces.

Results: Two to five renal segments were observed. Division into two segments, a cranial and a caudal, was the most common (42.62\%). The renal volume ranged from 101 to $173 \mathrm{~cm}(3)$ (mean $130.85 \mathrm{~cm}(3)$ ). The cranial segment was present in 39 of the 57 casts $(68.42 \%)$. It presented the greatest median value of proportional area $(50.00 \%)$ and also the greatest maximum value of proportional area, accounting for as much as $74.04 \%$ of the total kidney area. The ventral segment, which was found in 20 of the 57 casts (35.09\%), presented the lowest median value of proportional area $(13.87 \%)$ and showed the most variation in area (coefficient of variation $72.89 \%$ ). There was no significant statistical difference between the segmental areas as evaluated by Cavalieri's principle and by the point-counting planimetry method.

Conclusions: The distribution and size of the renal-arterial segments in pigs are not similar to those of the human kidneys. Therefore, this information must be taken into account by practitioners of urologic training or ablation using pigs as the animal model, as the structure of the porcine arterial segments cannot be transposed to humans.

\section{Editorial Comment}

The pig has been used as the favorite animal model for training and experimental research in urology, including many studies on laparoscopic total and partial nephrectomy, hemostasis techniques and more recently, 
ablative technologies, including radio frequency ablation and cryoablation. Therefore, a comprehensive knowledge on the proportional areas of the arterial segments would be important for evaluating the extension of experimental lesions in pigs. The aim of this study was to provide an analysis of the pig kidney segmentation and an analysis of the proportional area of each segment as measured on polyester resin endocasts of the kidney arterial vasculature.

The results demonstrated that the arterial segmental pattern in pigs are not similar to those of the human kidneys, and therefore, the experimental findings concerning renal ablative techniques using the pig must not be completely translated to clinical setting in humans.

Dr. Francisco J.B. Sampaio

Full-Professor and Chair, Urogenital Research Unit

State University of Rio de Janeiro

Rio de Janeiro, RJ, Brazil

E-mail: sampaio@urogenitalresearch.org

\section{Does benign prostatic hyperplasia originate from the peripheral zone of the prostate? A preliminary study}

Tang J, Yang JC, Zhang Y, Liu X, Zhang L, Wang Z, Li J, Luo Y, Xu J, Shi H

Department of Ultrasound, Chinese People's Liberation Army General Hospital, Beijing, PR China

BJU Int. 2007; 100: 1091-6

Objective: To compare the histological characteristics, cell proliferation, apoptosis and biological features in benign prostatic hyperplasia (BPH) in the peripheral (PZ) and transition zone (TZ) of the prostate.

Patients and Methods: Tissue from BPH in TZ and PZ was obtained from 68 patients undergoing transrectal ultrasonography-guided biopsy and used for both morphometric analysis and immunohistochemical studies. The epithelial, stromal and luminal composition of the tissue was determined using a computer-assisted method for quantitative morphometric analysis. Apoptosis was detected as the apoptotic index (AI) using the TdT dUTP nick-end labelling assay. Cell proliferation was determined as the proliferation index (PI) using Ki-67 immunostaining. The expression of epidermal growth factor receptor (EGFR), transforming growth factor beta1 (TGFbeta1), androgen receptor (AR) and bcl-2 were assessed immunohistochemically.

Results: There was no difference in the stroma/epithelium ratio between PZ and TZ hyperplastic nodules $(\mathrm{P}>$ 0.05). The mean AI in epithelium was almost identical to the corresponding PI. In stroma, no apoptotic cells were detectable. There was a significantly higher PI and AI in the glandular epithelial cells in PZ hyperplastic than in TZ hyperplastic nodules, but no difference in PI of the stromal cells between PZ and TZ hyperplastic nodules. There was significantly higher expression of TGFbeta1 and lower expression of EGFR and bcl-2 in PZ than TZ hyperplastic nodules $(\mathrm{P}<0.05)$. There was no difference in AR expression between $\mathrm{PZ}$ and $\mathrm{TZ}$ hyperplastic nodules $(\mathrm{P}>0.05)$.

Conclusions: These results indicate that some hyperplastic nodules in PZ might originate from the PZ, and the formation of these nodules might be modulated in a different way from that in the TZ.

\section{Editorial Comment}

It is well accepted that benign prostatic hyperplasia (BPH) develops from the transition zone (TZ) and from the periurethral glans, while carcinoma originates from the peripheral zone (PZ). Nevertheless, previous studies, including studies by that same group, reported that hypoechoic lesions in the PZ can be found as BPH 
histologically. Some authors suggested that the hyperplastic nodules in the PZ might be exophytic BPH in the TZ or the migration of ectopic TZ tissue into the PZ. The authors of the present study speculate that it is more likely that the hyperplastic nodules might originate from the PZ.

In the present study, the epithelial, stromal and luminal composition of the tissue was determined using a computer-assisted quantitative morphometric analysis method in biopsy specimens obtained from patients with $\mathrm{PZ}$ or TZ hypoechoic nodules on transrectal ultrasound, and that were histologically confirmed as BPH. The incidence of apoptosis and cell proliferation was analyzed comparatively in these hypoechoic nodules according to the zonal location. The authors also examined the relative expression of proteins involved in the regulation of prostate proliferation and apoptosis: (i) epidermal growth factor receptor (EGFR), which is in the signal transduction pathway that participates in the mediation of cell growth and has been implicated in prostatic epithelia cell proliferation in vitro; (ii) TGF $\beta 1$, the most extensively studied negative growth factor. The predominant effect of TGF $\beta 1$ on growth in vivo and in vitro is inhibition of cell proliferation; (iii) bcl-2, a potent apoptosis suppressor; and (iv) androgen receptor (AR); steroid binding to AR could stimulate proliferation and differentiation of epithelial cells and inhibit prostate cell apoptosis.

The results of the present study showed that no apoptotic cells were detectable in stroma, which indicated stromal growth due to cell proliferation, in the absence of cell death. The authors discussed that recent studies reported that the cell apoptotic rate in different regions of the ductal system is different, apoptosis being much less in the proximal ends. The proximal and distal regions of the ductal system correspond to the TZ and PZ of the human prostate. The present results showed that the cell apoptotic rate in the epithelium was much higher in PZ than in TZ hyperplastic nodules, which was in concordance with the higher TGF 1 and lower bcl-2 expression in the epithelium of PZ than TZ hyperplastic nodules. In the present study, TGF $\beta 1$ staining was intense in the epithelial cells, and bcl-2 expression was consistently restricted to the basal cell layer. This might explain why no apoptotic cells were detectable in the stroma of PZ and TZ hyperplastic nodules.

So, the authors concluded that the present results indicate that some hyperplastic nodules in the PZ might originate from the PZ, and the formation of these nodules might be modulated in a different way to that in the TZ.

\author{
Dr. Francisco J.B. Sampaio \\ Full-Professor and Chair, Urogenital Research Unit \\ State University of Rio de Janeiro \\ Rio de Janeiro, RJ, Brazil \\ E-mail:sampaio@urogenitalresearch.org
}

